\title{
Traumatische Querschnittlähmung
}

\section{Akutversorgung, Lähmungs- erstbehandlung und lebenslange Nachsorge nach Reitunfällen}

R. Flieger, Th. Meiners, Zentrum für Rückenmarkverletzte der Werner-Wicker-Klinik Bad Wildungen-Reinhardshausen

NOTFALLMEDIZIN 2003; 29: 296-303

Die traumatische Querschnittlähmung ist eine zwar seltene jedoch um so schwerere Folge eines Reitunfalles. Zwischen 1 und 4\% der frisch Querschnittgelähmten haben ihre Lähmung in Folge eines Reitunfalles. Das individuelle Risiko eines Reitsportlers, einen zu dieser Schädigung führenden Unfall zu erleiden, ist jedoch nicht zu beziffern. Die optimale Versorgung derart Betroffener beginnt mit adäquaten Maßnahmen am Unfallort und ist idealerweise so früh wie möglich an einem Querschnittzentrum fortzuführen, wo das Know-How zur Beherrschung lähmungstypischer Verläufe und Komplikationen vorhanden ist und zugleich auch die lebenslang erforderliche Nachsorge zu steuern ist.

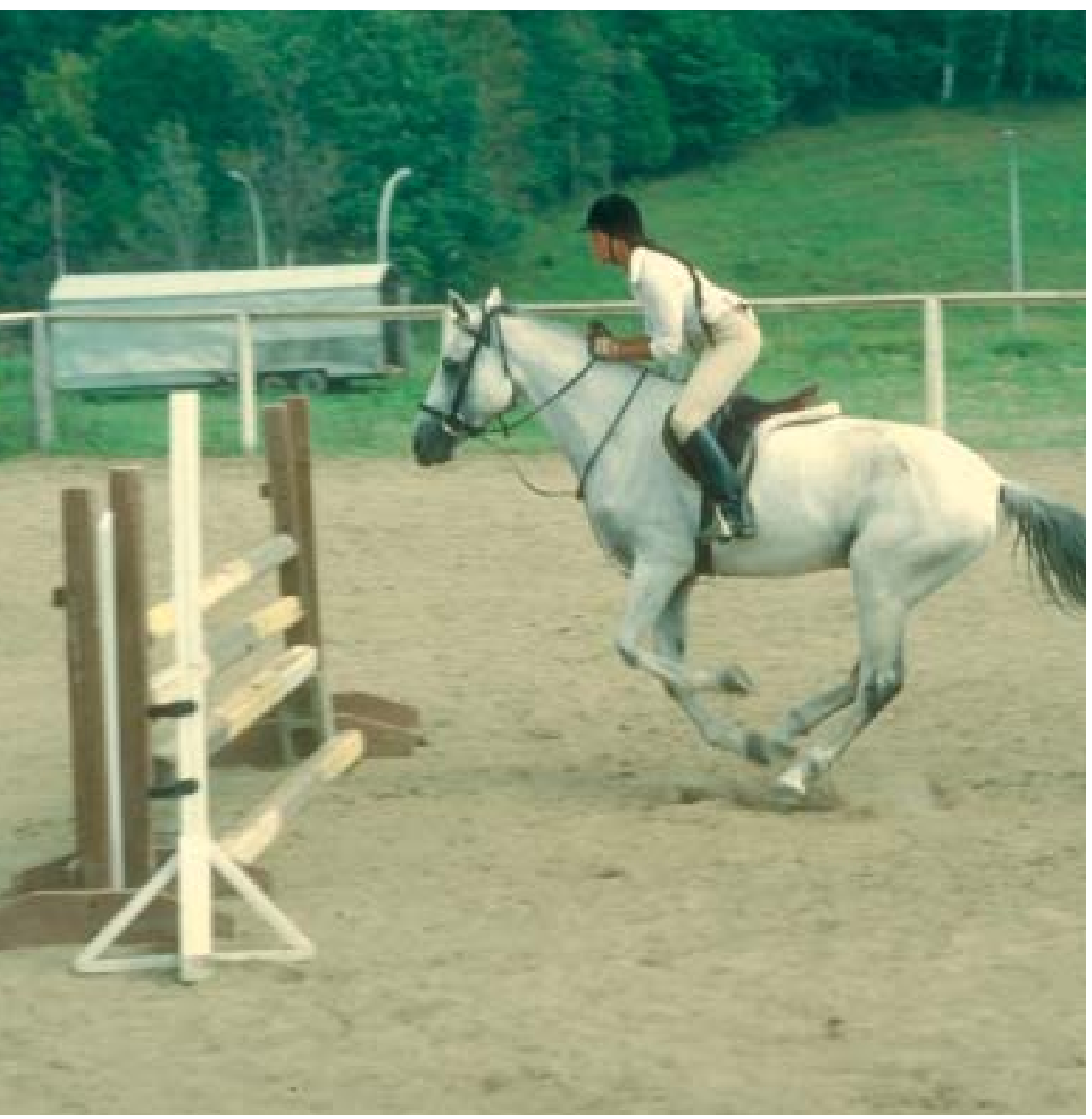

$\mathrm{S}$ eit dem spektakulären Reitunfall des Schauspielers Christopher Reeve im Jahre 1995, der nach hoher Halswirbelsäulenverletzung bis auf weiteres an einer Tetraplegie mit Beatmungsabhängigkeit leidet, ist die Querschnittlähmung und auch ihre Verursachung durch Reitunfälle wieder in das öffentliche Interesse gerückt. Zahlen, aus denen der (professionelle oder freizeitmäßige) Reitsportler sein Risiko, hierbei eine traumatische Querschnittlähmung zu erleiden, ableiten könnte, fehlen jedoch. Eine Studie der Orthopädischen Universitätsklinik Heidelberg, in der 1016 Patienten mit frischer traumatischer Rückenmarkschädigung in den Jahren 1985 bis 1997 zur Aufnahme gelangten, nennt hierunter ganze neun Fälle, die durch einen Reitunfall verursacht sind (11). Eine erst dieses Jahr publizierte Arbeit aus Australien, die Reitunfälle im Bundesstaat New South Wales in den Jahren 1976 bis 1996 auswertet, benennt (ohne Angabe der Gesamtfallzahl) retrospektiv 32 Fälle von traumatischer Querschnittlähmung durch Reitunfälle, die in den zwei Querschnittzentren des Bundesstaates behandelt wurden sowie 30 weitere Patienten im gleichen Zeitraum, die mit einer Wirbelsäulenverletzung durch Reitunfall ohne neurologischen Ausfall dort zur Aufnahme gelangten (10). Begleitverletzungen der Extremitäten oder innerer Organe waren häufig. Konkrete Maßnahmen zur Erhöhung der Sicherheit vor Wirbelsäulenverletzungen waren nicht abzuleiten, sondern lediglich die Empfehlung zu allgemeiner kontinuierlicher Sicherheits- 
erziehung für alle Reiter. Weitergehende Erkenntnisse vermag auch Silver aus einer retrospektiven Metaanalyse von drei britischen Querschnittzentren sowie elf Publikationen des anglo-amerikanischen Schriftums zum Thema nicht abzuleiten: Zwar kann er sagen, dass das Risiko, beim Reiten schwer verletzt zu werden, 20 mal so hoch ist wie beim Autofahren (eine schwere Verletzung je 350 Stunden Reiten gegenüber je 7000 Stunden Autofahren) und er differenziert weiter: eine schwere Verletzung je 100 Stunden freizeitmäßigen Reitens, eine auf fünf Stunden Springreiten von Amateuren und sogar eine schwere Verletzung auf nur eine Stunde Querfeldein-Reiten. Hinsichtlich der Inzidenz von Rückenmarkverletzungen durch Reitunfälle ist jedoch auch ihm nur die Aussage möglich, dass in den Jahren 1951 bis 1999 in den drei erfassten britischen Querschnittzentren bei zwischen 1,4 und 4,7\% der aufgenommenen Patienten mit frischer Querschnittlähmung diese auf einen Reitunfall zurückging (12).

Reitunfallopfer machen mithin nur einen kleinen Anteil unter den zur Zeit zirka 100000 Querschnittgelähmten aus, die in der Bundesrepublik Deutschland leben, jedes Jahr kommen etwa 1200 neue hinzu, wovon, wenn man die oben genannten Zahlen aus Heidelberg und Großbritannien extrapoliert, schätzungsweise ein bis vier Prozent durch Reitunfälle verursacht sein werden. Für die stationäre Behandlung aller dieser Menschen stehen bundesweit derzeit 1200 Betten in speziellen Querschnittzentren wie dem unsrigen zur Verfügung. Daraus ergibt sich zwangsläufig, dass eine gewisse „Dunkelziffer“ von querschnittgelähmten Patienten verbleibt, die sowohl zum Zeitpunkt des Lähmungseintritts als auch im weiteren Lebenslauf im Hinblick auf ihr Leiden suboptimal versorgt werden. Im Folgenden soll daher auch anschaulich gemacht werden, warum ein Querschnittgelähmter unbedingt in einem Querschnittzentrum oder, in speziellen Fällen, zumindest in Zusammenarbeit mit einem Querschnittzentrum betreut werden sollte.
Traumatische

Querschnittlähmung

Die Querschnittlähmung ist definiert als ein aus der Schädigung des Rückenmarksquerschnitts resultierendes Lähmungsbild mit Ausfall motorischer, sensibler und vegetativer Bahnen. Entsprechend ist nach Schädigungseintritt primär mit Funktionsstörungen aller drei Qualitäten des Nervensystems distal der Läsionshöhe zu rechnen (4).

Etwa 60\% aller Querschnittlähmungen treten ein als Folge einer wie auch immer gearteten oder ausgelösten - Verletzung, meistens einer Wirbelfraktur, seltener als alleinige Rückenmarksquetschung ohne knöcherne Verletzung (SCIWORA = Spinal Cord Injury Without Radiographic Abnormality, insbesondere bei Kindern und Jugendlichen (9)), wobei großenteils Verkehrs- und Arbeitsunfälle, zu kleinerem Teil auch Sportunfälle, Suizidversuche und die etwas beschönigend als „Badeunfälle“ bezeichneten Kopfsprünge in zu seichtes Wasser ursächlich sind. Die übrigen 40\% der Querschnittlähmungen verteilen sich auf angeborene Fälle infolge von Missbildungen der Wirbelsäule und des Rückenmarks, Erkrankungen (z.B. Tumoren, Infektionen und sonstige Entzündungen) und Durchblutungsstörungen und ihre Folgezustände (Spinalis-anterior-Syndrom). Letztere können ebenfalls in Ausnahmefällen traumatisch bedingt sein, beispielsweise durch Aortenruptur.

Die Ausdehnung der Lähmung am Körper ergibt sich aus der Höhe der Rückenmarksschädigung. Ist das Rückenmark in Höhe der Brust- bzw. Lendenwirbelsäule geschädigt, so kommt es zur Paraplegie. Der Begriff Tetraplegie bezeichnet eine Lähmung aller vier Extremitäten bei Läsion in Höhe der Halswirbelsäule. Im Einzelnen wird die Lähmungshöhe bezeichnet nach dem vom Kopf an gezählten letzten noch unversehrt funktionierenden Nervenwurzelsegment. Da das Rückenmark ja aus einer Vielzahl von Leitungsbahnen besteht, die im Rahmen einer der vorgenannten Schädigungen auch nicht unbedingt alle gleichermaßen betroffen sein müssen, kommen sowohl komplette als auch inkom- plette Lähmungen vor, die - je nachdem, welche Funktion betroffen ist zum Beispiel auch als „motorisch komplett und sensibel inkomplett“ bezeichnet werden. Bei der Motorik werden zwei Lähmungstypen unterschieden, nämlich die schlaffe Lähmung, wie sie auch bei allen Schädigungen von peripheren Nerven vorkommt und üblicherweise bei Wirbelverletzungen unterhalb $\mathrm{L} 2$. Hier sind motorische Aktivitäten der gelähmten Muskeln nicht auslösbar und die Muskeleigenreflexe sind vollkommen erloschen. Demgegenüber steht die spastische Lähmung, die mit einer elastischen Tonussteigerung der Muskulatur, Steigerung der muskulären Eigenreflexe und Automatismen bei erhaltenen intakten Rückenmarksanteilen unterhalb der Schädigung einhergeht. Bei vielen Querschnittlähmungen kommen Mischbilder beider Lähmungsformen vor, dadurch bedingt, dass das Rückenmark relativ kürzer ist als die Wirbelsäule und dadurch die Nervenwurzelursprünge im Rückenmark nicht mehr mit der Höhe der segmental zugehörigen Foramina der Wirbelsäule übereinstimmen.

\section{- Akutversorgung}

Im akuten frischen Zustand ist das komplette Querschnittsyndrom, gleich welcher Höhe, durch folgende Merkmale gekennzeichnet:

- komplette schlaffe Lähmung unterhalb des betroffenen Rückenmarkssegmentes,

- vollständige Lähmung der Blase (atone Überlaufblase) sowie von Darm und Potenz,

- vollständiger Sensibilitätsausfall für alle Qualitäten mit hyperalgetischer Randzone,

- Ausfall der Eigen- und Fremdreflexe sowie

- Ausfall der Gefäß- und Wärmeregulation.

Es besteht somit zunächst eine Störung nahezu aller entscheidender Steuerungsmechanismen des Organismus von der Läsionshöhe abwärts, die auch als spinaler Schock bezeichnet wird, so dass mit mittelbaren und unmittelbaren, nicht selten lebensbedrohlichen Komplikationen gerechnet werden 


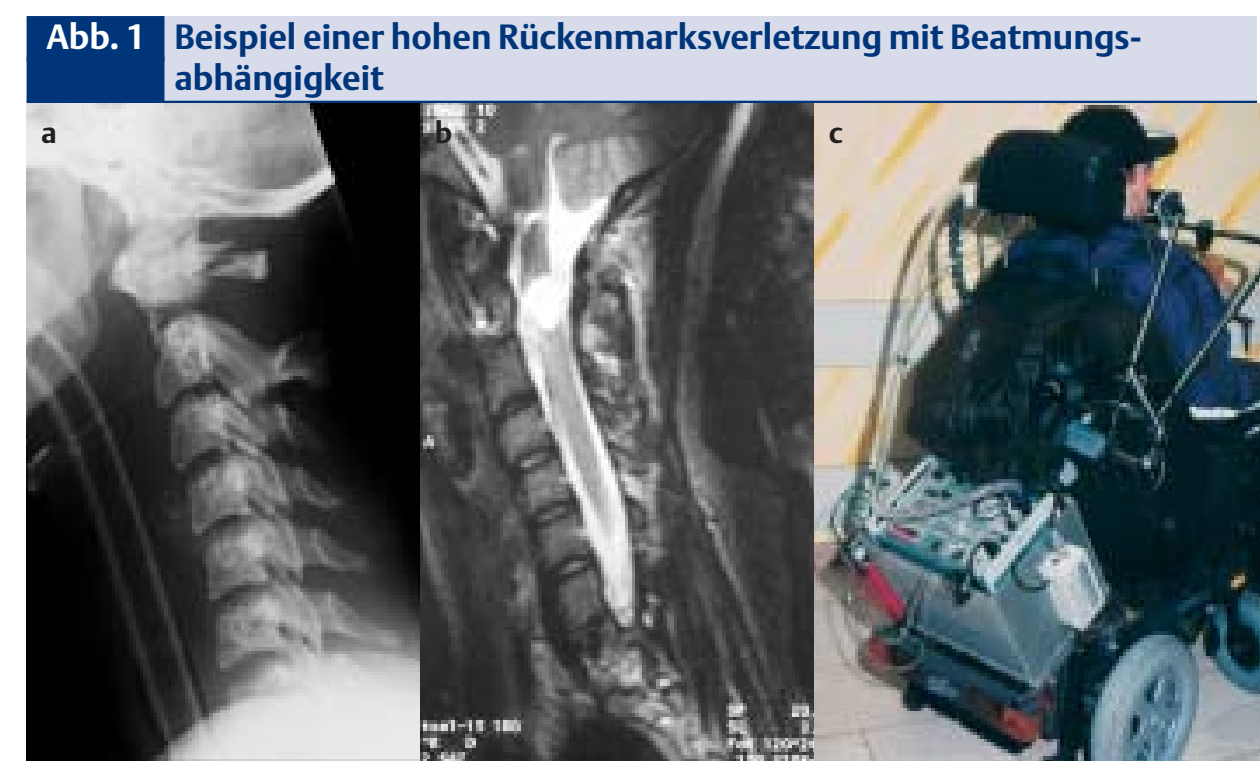

a) Fraktur des Dens axis und des Atlasrings b) im MRT sichtbare völlige Kontinuitätsunterbrechung des Myelons in gleicher Höhe c) Versorgung des Patienten mit E-Rollstuhl mit Kinnsteuerung und integriertem mobilem Beatmungssystem.

muss. Der genannte Ausfall der Gefäßregulation und die gleichzeitige Beeinträchtigung der Muskelpumpe bewirken einen Blutdruckabfall, bei entsprechender Verletzungshöhe kann auch eine Bradykardie bis hin zum Herzstillstand eintreten. Läsionen im Hals- und Brustwirbelsäulenbereich führen zu Problemen der Atmung, bei oberhalb des 3. Halswirbels gelegenen Schädigungen ist völliger Ausfall der Eigenatmung des Patienten die Folge, da die Wurzeln des N. phrenicus ( $\mathrm{C} 3$ bis C5) betroffen sind (Abb. 1). Insofern ist jede frische, insbesondere jede traumatisch entstandene Querschnittlähmung primär intensivpflichtig.

Im weiteren Verlauf, das heißt nach mehreren Tagen bis hin zu acht Wochen, ändert sich das Lähmungsbild auch bei kompletter Querschnittlähmung:

- je nach Läsionshöhe wird die Lähmung der Rumpf- und Extremitätenmuskulatur spastisch (bei Wirbelsäulenverletzung oberhalb L 1) und Reflexe treten wieder auf.

- Die Blasenlähmung stellt sich je nach Läsionshöhe um zur Reflexblase (bei Läsion oberhalb des Endabschnitts des Rückenmarks) oder zur inaktiven Blase (bei Läsion unterhalb des Endab-
Lähmung auch noch nach vielen Monaten erstaunliche Rückbildungstendenzen zeigen kann: Komplett bleibt komplett - Inkomplett wird inkompletter!

Die Akutversorgung eines traumatisch Querschnittgelähmten hat folgendermaßen zu erfolgen:

1. Am Unfallort selbst hat jeder Verdacht auf eine Wirbelsäulenverletzung dazu zu führen, dass der Patient wie ein Wirbelsäulenverletzter behandelt, das heißt geborgen und transportiert wird, um Rückenmarksschädigungen durch die Rettungsmaßnahmen zu vermeiden (Intubation, Helmabnehmen beim Motorradfahrer, Bergen Eingeklemmter usw.): Cervicalstütze, Vakuummatratze (Abb. $2)$, gegebenenfalls Hubschraubertransport. Wichtig ist eine möglichst umfassende Überprüfung des neurologischen Status durch den erstversorgenden Notarzt bereits am Unfallort, dessen Feststellungen den weiterbehandelnden Kollegen in der Klinik übermittelt werden müssen, wenn zum Beispiel wegen Intubation und Beatmung am Unfallort bei Klinikeinlieferung der neurologische Status nicht mehr überprüft werden kann. Die hochdosierte Kortikoidgabe

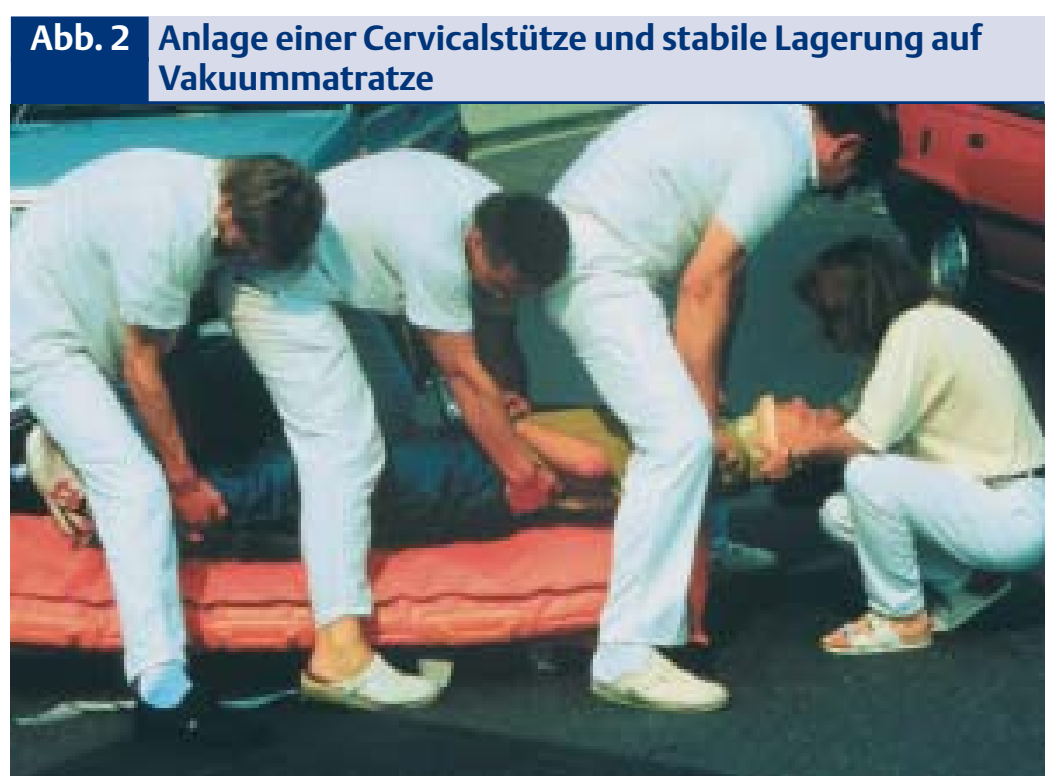

Jeder gestürzte Patient ist bis zum Beweis des Gegenteils wie ein Wirbelsäulenverletzter zu behandeln. 
noch am Unfallort, die auch innerhalb der ersten 24 Stunden nach dem Trauma in der Klinik fortzusetzen ist, ist die einzige medikamentöse Maßnahme, deren Auswirkung auf den neurologischen Outcome des Traumas erwiesen ist und daher (allerdings nicht unumstrittenen) notfallmedizinischen Standard darstellt $(2,3,5)$.

2. In der Klinik hat nach weiterer Sicherung der Vitalfunktionen, das heißt von Atmung und Kreislauf, die vollständige Diagnostik der Läsion zu erfolgen (körperlicher und neurologischer Befund, Röntgen, CT, ggf. Kernspintomographie). Bei Halswirbelsäulenverletzungen wird eine Haloextension angelegt zur Reposition bzw. Retention der Halswirbelsäule und damit Druckentlastung des Rückenmarks (Abb. 3).

3. Wie bereits erwähnt, bedarf jeder frische, insbesondere traumatisch Querschnittgelähmte, der intensivmedizinischen Versorgung.

4. Die Frakturbehandlung der Wirbelsäule erfolgt in der Mehrzahl der Fälle operativ, die konservative Frakturbehandlung ist heutzutage im Wesentlichen nur noch stabilen Frakturformen ohne wesentliche Stellungsabweichung vorbehalten. Der Operationszeitpunkt ist mit Rücksicht auf die Schwere des Traumas und auf den neurologischen Status zu wählen, bei eintretender neurologischer Verschlechterung im Verlauf besteht dringliche Indikation. Insbesondere bei am Unfallort bereits bestehender kompletter Lähmung ist mit einer Einflussnahme auf den neurologischen Endzustand nicht zu rechnen, daher ist das Abwarten einer Besserung des Allgemeinzustandes beim Verletzten legitim. Die Operationsindikation ergibt sich im Übrigen wie bei Wirbelsäulenfrakturen ohne Lähmung, das heißt aus Gründen der Stabilität und Stellung der Wirbelsäule. In diesen Fällen wird die Operation dann nach Ablauf eines Intervalls vorgenommen, im Bereich der Rumpfwirbelsäule gegebenenfalls auch zweizeitig von dorsal transpedikulär und von ventral intersomatisch (Abb. 4). In dieser Phase finden auch weitere operative Maßnahmen wegen an anderen Körperteilen bestehender Begleitverletzungen statt, gegebenenfalls auch schon vorgängig zur Wirbelsäulenstabilisierung, wenn es aus vitaler Indikation erforderlich ist (z.B. Versorgung einer Milzruptur oder einer offenen Extremitätenfraktur).

5. Der genannten Phase der Operationen, die in der Mehrzahl der Fälle noch auf der Intensivstation stattfindet, schließt sich wenn möglich - auf der peripheren Station des Querschnittzentrums, die Phase der eigentlichen Querschnitterstbehandlung an. Ein Teil der Patienten ist zuvor in Allgemeinkliniken, insbesondere unfallchirurgischen oder neurochirurgischen Kliniken, vorbehandelt worden und wird erst für diesen Abschnitt in das Querschnittzentrum verlegt. Aufgrund der personellen und technischen Ausstattung ist unser Zentrum jedoch in der Lage, auch frischverletzte Patienten direkt vom Unfallort kommend aufzunehmen. In Zusammenarbeit aller Fachabteilungen des Hauses können neben der Wirbelsäulenverletzung und Querschnittlähmung auch alle üblichen Begleitverletzungen ad-

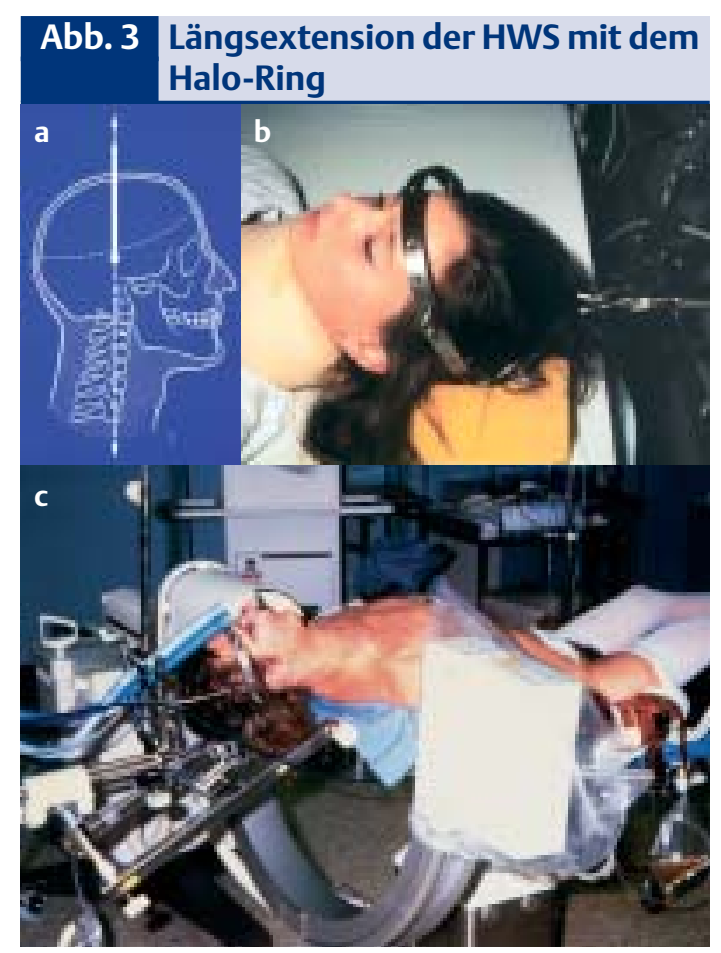

a) Durch Längsextension der HWS mit dem HaloRing wird das Alignement der HWS ausgerichtet. b) und c) Die Extension erfolgt im Rahmen der OP-Vorbereitung, kann aber auch als konservatives Verfahren (mit Halo-Body-Jacket kombiniert) fortgeführt werden, bei Querschnittpatienten nur in Ausnahmefällen.

äquat versorgt werden. Auch nicht unfallbedingt querschnittgelähmte Patienten kommen üblicherweise in dieser Phase in das Querschnittzentrum, nachdem ihre zur Lähmung führende Grunderkrankung diagnostiziert und behandelt worden ist.

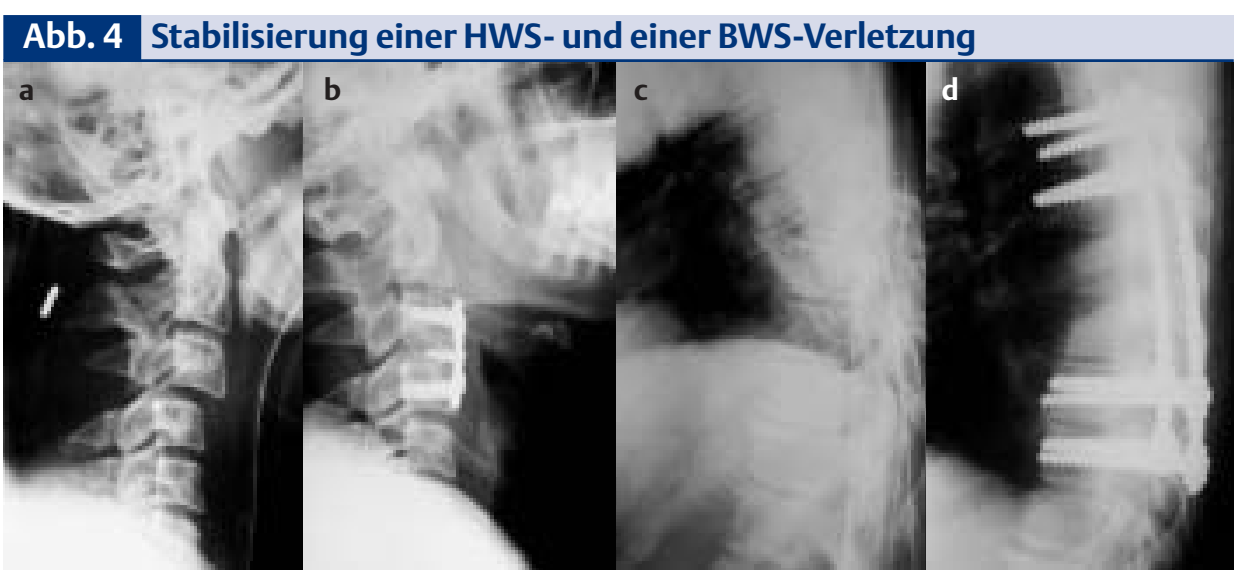

a) HWS-Verletzung (Luxationsfraktur C3/C4) präoperativ b) postoperativ: Stabilisierung durch ventrale Spondylodese mit Beckenkammspan und Platte c) BWS-Verletzung (BWK 6/7-Fraktur) präoperativ d) postoperativ: Stabilisierung durch Fixateur interne von dorsal. 


\section{Abb. 5 Sacrales Decubitalulcus bei thorakaler Paraplegie}

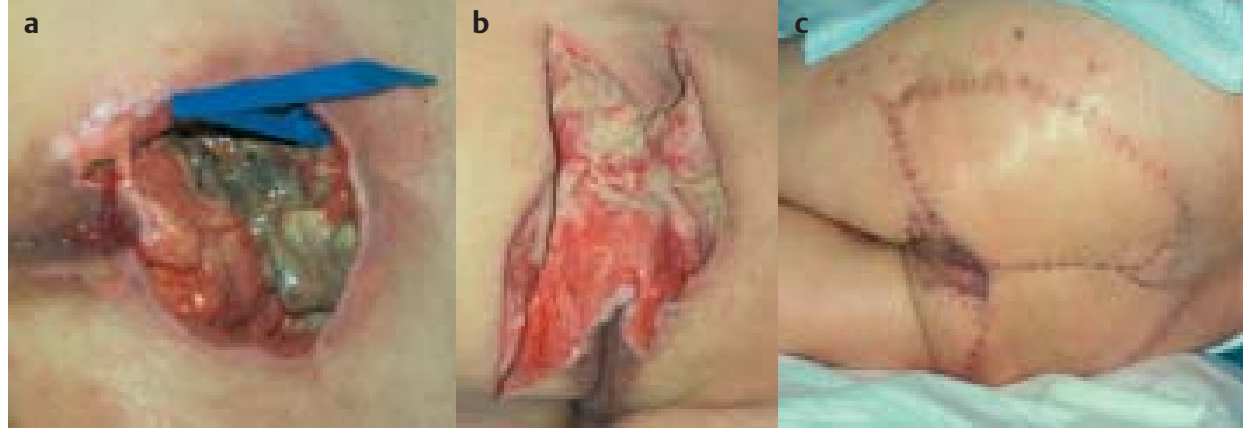

a

a) Aufnahmebefund mit tiefen Nekrosen und Taschenbildung, b) Befund nach operativem Debridement und Wundgrundkonditionierung c) Befund nach Deckung durch beidseitige Glutaeus-Maximus-Lappenplastik. Zur Vermeidung der Stuhlkontamination des analnah gelegenen Wundgebietes wurde hier eine Sigmakolostomie angelegt, die auf Wunsch des Patienten belassen wurde.

\section{Lähmungsspezifische Erstbehandlung} Ziele der Querschnitterstbehandlung sind:

- Stabilisation

- Mobilisation

- Komplikationsmanagement und

- Hilfe zur Selbsthilfe.

Hieran sind alle im unmittelbaren Patientenkontakt stehenden Berufsgruppen im Sinne der „comprehensive care“ beteiligt, nämlich Ärzte (Orthopäden, Chirurgen, Urologen), Pflege, Physio- und Ergotherapeuten, Psychologen, Sozialarbeiter und Orthopädietechniker. Nach Stabilisierung steht die Mobilisierung des Patienten unter Ausschöpfung aller noch vorhandener Funktionen ober- und unterhalb seiner Rückenmarkläsionshöhe mit dem Ziel der Wiedereingliederung in den privaten und möglichst auch den beruflichen Bereich an. Durch Gestellung individuell anzupassender Hilfsmittel vom Rollstuhl über ergotherapeutisch gefertigte Esshilfen bis zum behinderungsgerecht ausgerüsteten PKW ist für den Betroffenen weitestmögliche Unabhängigkeit von der Fremdbestimmung durch Hilfspersonen anzustreben.

Sämtliche genannten Berufsgruppen sind bereits auch von der Intensivtherapiephase an in die Behandlung des Querschnittgelähmten mit eingebunden. Einen besonderen Schwerpunkt dieser Bemühungen stellt die von Nichtbetroffenen nicht in ihrer Bedeutung zu erfassende
Regulierung der Blasen- und Mastdarmentleerung dar, deren lähmungstypadäquate Technik im Rahmen der Erstbehandlung erarbeitet und im Weiteren vom Patienten selbst und auch von seinen ihn häuslich versorgenden Personen erlernt und praktiziert wird.

Die durch gebündelten Einsatz aller genannten Kräfte erreichbaren Ziele sind jedoch sehr unterschiedlich und hängen im Wesentlichen vom Lähmungsausmaß und der Lähmungshöhe ab, aber auch von Begleitumständen wie Lebensalter des betroffenen Patienten, Konstitution und inneren Begleiterkrankungen und natürlich der Motivation des Betroffenen. Diese Umstände finden naturgemäß auch Eingang in die stationäre Behandlungsdauer der Patienten. In der Regel kann man davon ausgehen, dass die unkompliziert verlaufene Querschnitterstbehandlung eines Paraplegikers, etwa sechs Monate in Anspruch nimmt, die Erstbehandlung eines Tetraplegikers dauert häufig bis zu zwölf Monaten, in Einzelfällen auch darüber. Insbesondere bei inkompletten Lähmungsbildern, bei denen es im Laufe der Behandlungszeit auch noch zur Erholung primär ausgefallener Funktionen kommt, kann zur therapeutischen Erarbeitung dieser hinzugekommenen Funktion und damit zur Verbesserung der funktionellen Ausgangsposition des Patienten bei Entlassung von diesen groben Zeitvorgaben auch nach oben abgewichen werden.

\section{Lebenslange Nachsorge}

Die Zuständigkeit eines Spezialzentrums für den Querschnittgelähmten endet jedoch nicht mit der Entlassung aus der Erstbehandlung, sondern ist lebenslang weitergegeben, da es infolge der Querschnittlähmung zu spezifischen Komplikationen kommen kann, die mit den Mitteln eines Allgemeinkrankenhauses üblicherweise nicht adäquat zu versorgen sind, deren Entstehung vielmehr im Einzelfalle in einem in der Behandlung von Querschnittgelähmten unkundigen Krankenhaus sogar befürchtet werden muss. Von den 100 Betten des hiesigen Zentrums für Rückenmarkverletzte sind ständig gut 50\% mit Patienten belegt, deren stationäre Wiederaufnahme wegen Komplikationen oder funktioneller Verschlechterung erforderlich wurde.

\section{Decubitus}

An erster Stelle ist hier der Decubitus oder das Druckgeschwür zu nennen. Hiervon betroffene Patienten machen regelmäßig zirka 25\% der Belegung der Gesamtabteilung aus. Ein Decubitus entsteht bekanntermaßen als Folge einer lokalen Minderdurchblutung der Körperoberfläche, verursacht durch äußere Druckeinwirkung und auch Scherkräfte. Chemische Noxen, wie zum Beispiel Schweiß und Exkremente bei unkontrollierter Entleerung, tragen ein Übriges zur Schadensentstehung bei.

Am leichtesten ist der Decubitus zu behandeln, den man gar nicht erst bekommt. Da die Ursachen bekannt sind, gilt es, den Druck zu vermeiden, wo immer er entsteht. Wichtig ist die regelmäßige Kontrolle der gefährdeten Körperpartien hinsichtlich druckbedingter Rötungen und die Vermeidung von druckgefährdenden Momenten. Dies lernen die Patienten im Rahmen der Querschnitterstbehandlung selbst durchzuführen, soweit ihre Lähmungshöhe das erlaubt.

\section{Tieferes Druckgeschwür}

Das tiefere Druckgeschwür ist, soweit erforderlich, chirurgisch oder durch entsprechende äußerlich anzuwendende Präparate zu behan- 
deln und im weiteren Verlauf bei geeigneten Defekten durch eine entsprechende Verbandsbehandlung, in ausgedehnteren Fällen durch operative Maßnahmen zur Ausheilung zu bringen, die ab einer gewissen Defektgröße und -tiefe bevorzugt werden. Es handelt sich um standardisierte plastisch-chirurgische Techniken, die bei komplikationslosem postoperativen Verlauf ab der dritten postoperativen Woche beginnend einen schrittweisen Belastungsaufbau der gedeckten Region zulassen (7, Abb. 5). Mit der erfolgreichen Behandlung des Decubitalulcus ist das Problem jedoch nicht gelöst, sondern es muss abgeklärt werden, wodurch das aktuelle Decubitalulcus entstanden ist. Nicht immer ist die Nachlässigkeit des Patienten bei der Entlastung anzuschuldigen, es können auch medizinische Gründe bestehen, die zu einer Veränderung der Sitzposition mit besonderer Belastung prominenter Knochenpunkte führen können, beispielsweise ein Beckenschiefstand bei eingetretener Lähmungsskoliose. Zur Erfassung derartiger Problemfelder im Bereich der Gesäßregion wird in unserer Abteilung ein Sitzflächendruckmesssystem eingesetzt, welches besonders

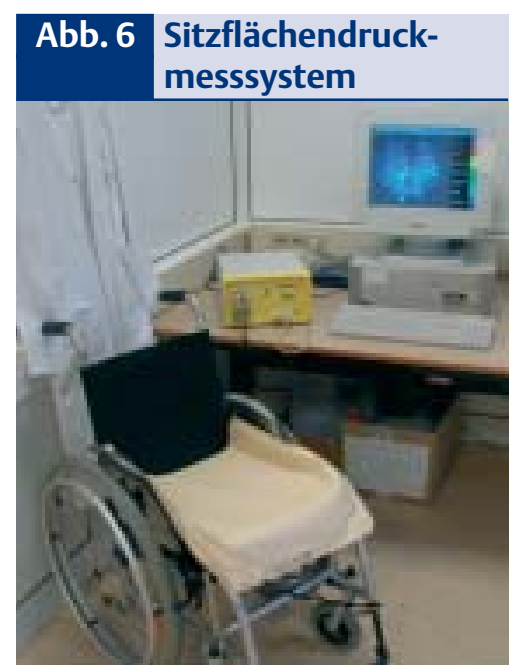

Mit diesem System können die Druckverhältnisse im Bereich der Sitzfläche des Querschnittgelähmten objektiviert werden und damit die Eignung bestimmter Sitzkissen geprüft, aber auch Hinweise auf das Vorliegen sonstiger Decubitusursachen abgeklärt werden. exponierte Druckpunkte erfasst und auf diese Weise Decubitusursachen aufspüren hilft (Abb. 6).

\section{Spastik}

Spastik ist ein weiterer Grund für stationäre Wiederaufnahmen Querschnittgelähmter im Zentrum für Rückenmarksverletzte. Positiver Effekt der Spastik ist das geringere Risiko von Muskelschwund und Thrombose. Häufig ist die Spastik aber störend bis quälend, so dass sie den betroffenen Patienten beim Sitzen und bei seiner Selbsthilfefähigkeit beeinträchtigt. In leichten Fällen ist, wie auch bereits im Rahmen der Erstbehandlung, intensive regelmäßige Krankengymnastik im Sinne der Muskeldehnung und auch eigentätiges Durchbewegen ausreichend, unterstützt durch physikalische Therapie und Sauna. In der Mehrzahl der Fälle kommen oral einzunehmende spastiklösende Medikamente wie Baclofen oder Dantrolen zum Einsatz. Wenn auch die oral einzunehmende Höchstdosis dieser Präparate keine funktionell ausreichende Spastikdämpfung bewirkt, ist die Gabe des Präparates Baclofen auch direkt an das Rückenmark über einen dort eingelegten Katheter möglich, der über eine im Bereich der Bauchdecke subkutan implantierte Pumpe gespeist wird. Die Pumpe setzt das Präparat kontinuierlich frei und muss in regelmäßigen Abständen, je nach Präparatebedarf, ambulant wieder aufgefüllt werden. Die Implantation derartiger Pumpensysteme erfolgt im Querschnittzentrum nach entsprechender Vortestung.

Bei bereits länger bestehender überschießender Spastik treten Kontrakturen ein, sodass zum Beispiel der Fuß in Spitzfußstellung fixiert ist und nicht mehr durch krankengymnastische Maßnahmen und ähnliches in die Neutralstellung gebracht werden kann. Der Versuch, durch Standbelastung im Stehgerät wieder zu einem plantigraden Auftritt zu kommen, hat eher eine $\mathrm{Zu}$ nahme der spastischen Kontraktur zur Folge. In solchen Fällen sind Eingriffe zur Sehnenverlängerung beziehungsweise Sehnendurchtrennung indiziert, um hier zu einer

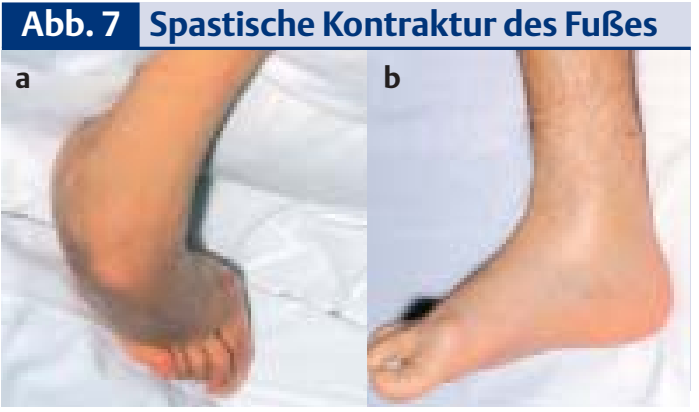

a) spastische Kontraktur des Fußes im Sinne des Pes equinovarus b) operatives Korrekturergebnis nach HOKE-Tenotomie der Achillessehne, Zehenbeugertenotomien und Teiltransfer der Tibialisanterior-Sehne auf die Basis des 5. Mittelfußknochens.

funktionellen Verbesserung für den Patienten zu kommen (6, Abb. 7).

\section{Paraosteoarthropathie}

Die Paraosteoarthropathie (POA) ist ein Krankheitsbild, bei dem es in der unmittelbaren Umgebung von großen Gelenken zu einer Weichteilossifikation kommt, die das Gelenk bis hin zur völligen funktionellen Versteifung überbrückt, ohne es jedoch selbst dabei in die Verknöcherung einzubeziehen. Derartiges kommt nicht selten bei Querschnittlähmungen, aber auch bei SchädelHirntrauma-Patienten und bei polytraumatisierten langzeitbeatmeten Patienten vor. Am häufigsten hiervon betroffen sind die Hüftgelenke. Die einzig mögliche Behandlung ist die operative Entfernung der Ossifikation, die jedoch erst dann vorgenommen werden sollte, wenn die Verknöcherung „ausgereift“ ist, da sonst mit einem noch schlimmeren Wiederauftreten zu rechnen ist. Das aktuell anerkannte Behandlungsregime in solchen Fällen umfasst neben der POA-Resektion eine im Zeitraum von 24 Stunden vor bis 24 Stunden nach dem Eingriff vorzunehmende single-shot-Bestrahlung (an der Hüfte mit $7 \mathrm{~Gy}$ ) sowie die orale Gabe eines oralen Antirheumatikums für drei Wochen zur Prophylaxe der Reossifikation (1). Die Einsteifung eines Hüftgelenkes, insbesondere in weitgehender Streckstellung, beeinträchtigt den Patienten erheblich bei der Sitzposition im Rollstuhl, macht ihn, zum Beispiel beim Umsetzen und auch beim 
An- und Ausziehen vermehrt von fremder Hilfe abhängig und stellt aufgrund der ungünstigen Sitzposition auch ein vermehrtes Decubitusrisiko dar, so dass der Patient von einer erfolgreichen POA-Entfernung in jedem Falle profitiert. Da ein derartiger Eingriff im Bereich des Hüftgelenkes mit einem nicht unerheblichen Blutverlust verbunden sein kann, und es sich um einen planbaren Wahleingriff handelt, wird dem Patienten an hiesiger Klinik üblicherweise die Möglichkeit der präoperativen Eigenblutspende angeboten.

\section{Verschlechterung im Lähmungs- status}

Ein großer Teil der Wiederaufnahmepatienten kommt zur stationären Behandlung wegen im Verlauf eingetretener funktioneller Defizite. Dazu zählen Verschlechterungen im Lähmungsstatus, die im vollen Umfange abzuklären sind, da unter Umständen sogar operative Maßnahmen zu ihrer Beseitigung unternommen werden müssen, Kraftund Selbständigkeitsverluste nach zu Hause durchgemachten Phasen der Immobilität (z.B. nach fiebrigen Infekten) oder auch Verschlechterungen, die sich aus dem zunehmenden Lebensalter oder aus allgemeinen Begleiterkrankungen ergeben. Diesen Änderungen ist neben der Abklärung durch entsprechende Physio- und Ergotherapie unter stationären Bedingungen Rechnung zu tragen, wobei in Einzelfällen die festzustellende funktionelle Verschlechterung auf Dauer auch hingenommen werden muss und die Ausstattung des betroffenen Patienten mit Hilfsmitteln und gegebenenfalls pflegerischen Leistungen der neuen Situation anzupassen ist. In der Mehrzahl der Fälle gelingt es, durch die stationäre Maßnahme den funktionellen Zustand des Patienten wieder soweit zu verbessern, wie er nach Abschluss der Erstbehandlung war, in Einzelfällen, insbesondere wie erwähnt, bei inkompletten Lähmungen, ist es sogar möglich, dass auch hinzugewonnene Funktionen physiotherapeutisch aufgearbeitet werden können, so dass ein weiterer funktioneller Gewinn für den Pa- tienten entsteht. In Einzelfällen muss jedoch auch beispielsweise in höherem Lebensalter, eine funktionelle Verschlechterung zur Kenntnis genommen werden und die Hilfsmittel- und Pflegeausstattung des Patienten auf die neue Situation abgestimmt werden, zum Beispiel nach abgelaufenem Herzinfarkt, nach koronarem Bypass oder ähnlichen Veränderungen der Belastbarkeit des Patienten.

\section{Osteopenie mit vermehrter Frakturanfälligkeit}

Das bei den meisten Querschnittgelähmten nicht oder nur noch im Rahmen der Therapie stattfindende Stehen und Gehen hat zur Folge, dass es insbesondere im Bereich der unteren Extremitäten dieser Patienten zu einer Osteopenie mit vermehrter Frakturanfälligkeit kommt. Dementsprechend kommt es bei Querschnittgelähmten auch nicht selten bei eigentlich nicht adäquater Gewalteinwirkung zu Frakturen im Bereich der unteren Gliedmaßen, die teilweise auch verspätet diagnostiziert werden. Wegen des Decubitusrisikos kommt eine Gipsbehandlung derartiger Frakturen grundsätzlich nicht in Betracht, sondern es sind in der Regel operative Maßnahmen erforderlich, die in den Standardtechniken der operativen Frakturbehandlung vorgenommen werden, jedoch der besonderen Knochenbeschaffenheit des Querschnittgelähmten Rechnung zu tragen haben. Insbesondere am Unterschenkel setzen wir gerne den RingFixateur ein, da dieses Verfahren den betroffenen Knochen nur geringstmöglich traumatisiert und trotzdem bereits kurz nach der Operation eine Belastung der Extremität zulässt, die die Frakturheilung beschleunigt (8).

\section{Probleme der Blase und des Mastdarms}

Ein wesentlicher Wiederaufnahmegrund bei unseren Querschnittpatienten sind auch Probleme mit der Blase und dem Mastdarm. Im Rahmen der Querschnitterstbehandlung werden die Patienten nach entsprechender Diagnostik auf ein geeignetes Verfahren der Entleerung der Blase und des Mastdarmes ein- gestellt, welches sie, soweit aufgrund der Lähmungshöhe möglich, auch selbst erlernen und zu Hause selbst anwenden sollen. Durch Veränderungen der Spastiksituation des Beckenbodens kann es jedoch dazu kommen, dass die bisher praktizierte Technik der Blasen- und Darmentleerung nicht mehr adäquat funktioniert, dass zum Beispiel die urologischerseits verordneten Medikamente zur Unterdrückung der Blasenspastik keine ausreichende Wirkung mehr zeigten, so dass es zu zwischenzeitlicher Enuresis kommt, dass gehäuft Harnwegsinfekte auftreten oder aber die Restharnmengen ansteigen. Entsprechende diagnostische Verfahren, wie sie der Urologie in Form der Blasenmanometrie und entsprechenden Durchleuchtungstechniken mit Kontrastmittelanwendung (so genannte Videourodynamik) zur Verfügung stehen, sind für die Entleerung des Darmes zur Zeit noch nicht hinreichend etabliert, so dass zur Wahl eines geeigneten Verfahrens zur Darmentleerung derzeit noch auf allgemeine Erfahrungen zurückgegriffen wird. Die im Rahmen der allgemeinen Proktologie zur anorektalen Funktionsdiagnostik etablierten Verfahren - wie anale und rektale Manometrie, Transitzeitbestimmung mit röntgenologisch sichtbaren Markern u.ä. - sind hinsichtlich ihrer Aussagefähigkeit für den lähmungsbetroffenen Darm noch nicht validiert, so dass wir aus diesen Befunden noch nicht so sichere therapeutische Konsequenzen wie bei der Blasenentleerung des gelähmten Patienten ziehen können.

\section{Summary}

Traumatic paraplegia is a rare but very serious consequence of a horseriding accident which accounts for between 1 and 4\% of fresh paraplegias. The risk of an individual rider's having an accident resulting in such an injury is, however impossible to determine. Optimal care of victims begins with the appropriate measures undertaken at the site of the accident and, ideally should be continues as early as possible in a specialized center for paraplegia having the ne- 
cessary know-how and skills to cope with the typical sequelae of paresis and complications, and where aftercare (life-long) can be coordinated.

\section{Literatur}

1. Abel R, Meiners T, Gerner HJ. Die Resektion von heterotopen Ossifikationen des Hüftgelenks Querschnittgelähmter. Operat Orthop Traumatol 2002; 14 (1): 16-28

2. Bracken MB, Shepard M] et al. A randomized controlled trial of methylprednisolone or naloxone in the treatment of acute spinal-cord injury. Results of the Second $\mathrm{Na}$ tional Acute Spinal Cord Injury Study. N Engl J Med 1990; 322 (20): 1405-1411

3. Bracken MB, Shepard MJ et al. Methylprednisolone or tirilazad mesylate administration after acute spinal cord injury: 1 -year follow up. Results of the third National Acute Spinal Cord Injury randomized trial. J Neurosurg 1998; 89 (5): 699-706

4. Gerner HJ. Die Querschnittlähmung. Berlin, Blackwell Wissenschaft 1992

5. Hugenholtz H, Cass DE et al. High-dose methylprednisolone for acute closed spinal cord injury: only a treatment option. Can J Neurol Sci 2002; 29 (3): 227-235

6. Keenan MA, Kozin SH, Berlet AC. Manual of orthopaedic surgery for spasticity. New York: Raven Press, 1993

7. Lüscher NJ. Dekubitalulzera der Beckenregion: Diagnostik und chirurgische Therapie. Bern: Verlag Hans Huber, 1989

8. Meiners $\mathrm{T}$, Keil $\mathrm{M}$ et al. Use of the ring fixator in the treatment of fractures of the lower extremity in long-term paraplegic and tetraplegic patients. Spinal Cord 2003; 41 (3): 172-177

9. Pang D, Wilberger JE. Spinal cord injury without radiographic abnormalities in children. J Neurosurg 1982; 57 (7): 114-129 10. Roe JP, Taylor TKF et al. Spinal and spinal cord injuries in horse riding: The New South Wales experience 1976-1996. ANZ J Surg 2003; 73 (5): 331-334

11. Schmitt H, Gerner HJ. Paralysis from sport and diving accindents. Clin J Sport Med. 2001; 11 (1): 17-22

12. Silver JR. Spinal injuries resulting from horse riding accidents. Spinal Cord 2002; 40 (6): 264- 271

\section{Anschrift für die Verfasser}

Dr. med. Robert Flieger

Werner-Wicker-Klinik

Kreuzfeld 4

34537 Bad Wildungen-Reinhardshausen

\section{Buch}

\section{Unfallchirurgische Behandlung Paradebeispiel Unterschenkelbruch}

Szyszkowitz R (Hrsg.). Tscherne Unfallchirurgie. Unterschenkel. Heidelberg: Springer-Verlag, 2003

240 Seiten, 352 Abbildungen, 5 Tabellen, ISBN 3-540-63284-0, 139,95 Euro

Der Unterschenkelbruch ist seit Jahrhunderten ein Paradebeispiel der unfallchirurgischen Behandlung beziehungsweise ihres Fortschrittes, da er nicht nur häufig ist, sondern auch - besonders der offene - häufig mit Komplikationen assoziiert war und die Lebensqualität des Verunglückten beeinflusst. Aber auch die verzögerte Heilung und deren Behandlung ruft wegen des nur teilweise vorhandenen zirkulären Muskelmantels und der relativ schlechten Durchblutung am körperfernen Unterschenkel - auch beim isolierten Trauma - noch immer Probleme hervor.

Der vorliegende Band der Reihe „Tscherne Unfallchirurgie“ gliedert sich in sieben Kapitel: Geschlossene Schien- und Wadenbeinbrüche, Offene Schenkelbrüche, distale intraartikuläre Unterschenkelbrüche, Sprunggelenkfrakturen, Bandverletzungen des oberen Sprunggelenks, posttraumatische Fehlstellungen am Unterschenkel, aseptische und septische Pseudarthrosen am Unterschenkelschaft. Innerhalb der einzelnen Kapitel zieht sich eine gewisse Reihenfolge wie ein roter Faden bis zum Ende durch das Buch so kann man in jedem Kapitel das Gewünschte auch ohne Zuhilfenahme des ausführlichen Sachregisters respektive Inhaltverzeichnisses schnell finden. Jedes dieser Kapitel endet mit einer umfangreichen Literatursammlung, die dem Interessierten den Weg zum weitergehenden Studium ebnet. Über 350 Zeichnungen und Abbildungen erleichtern das Verständnis des komplexen Stoffes. Schnell hat man sich bei der einen oder anderen Textstelle festgelesen. Allerdings ist das Schriftbild ein wenig (zu) klein geraten - langes Lesen strengt in Laufe der Zeit an. Der Text kommt einem nie zu lang vor. Der Autor holt nicht weit aus und schweift nicht vom Thema ab, sondern bringt das Wesentliche verständlich auf den Punkt - wenn auch mancher Satz in zwei oder drei Teile aufgeteilt werden könnte.

In dieser Auflage wurden einige Frakturen anderen Bänden zugeordnet (Verletzungen des Kniegelenkes und des angrenzenden Tibiakopfes finden sich im Band „Knie“, Verletzungen des Sprungbeines und die Verletzungen distal davon im Band „Fuß“), aber auch aus anderen Bänden übernommen (Verletzungen des oberen Sprunggelenkes einschließlich von Verletzungen der Knöchel, lokaler Sehnen und Bänder). Eingehend sind die Vor- und Nachteile der Alternativen bezüglich der Therapie beschrieben, aber auch das Komplikationsmanagement kommt in den letzten Kapiteln dieses Bandes ausführlich zur Darstellung.

Ergänzungen können sowohl hinsichtlich Diagnostik und Behandlung (z.B. Spongiosaplastik, Sehnennähte), als auch bezüglich der Komplikationen (z.B. Infektion, Pseudarthrose) in den anderen Bänden gefunden werden. Zahlreiche Tipps und Tricks - von der Lagerung bis zu den Zugängen und Operationstechniken - werden erwähnt und diverse Behandlungsschritte ausführlich illustriert.

Das Buch ist für den angehenden (Unfall-) Chirurgen oder Orthopäden wärmstens zu empfehlen.

Dr. Ingo Blank, Weil im Schönbuch 